\title{
Almost sure oscillation of certain random processes
}

\author{
JEAN-MARCAZAÏS ${ }^{1 *}$ and MARIO WSCHEBOR ${ }^{2}$ \\ ${ }^{1}$ Laboratoire de Statistique et Probabilités, URA-CNRS D0745 Université Paul Sabatier, \\ 31062 Toulouse, France \\ ${ }^{2}$ Centro de Matemática, Facultad de Ciencias, Universidad de la República, Montevideo, Uruguay
}

We show that for various classes of stochastic process, namely Gaussian processes, stable Lévy processes and Brownian martingales, we have almost sure weak convergence of the oscillation in the measure space $([0,1], \lambda), \lambda$ being Lebesgue measure. This result is used to obtain almost sure weak approximation of the occupation measure via numbers of crossings.

Keywords: crossings of a level; Gaussian processes; martingales; occupation measure; stable processes

\section{Introduction}

Let $X=\left\{X_{t}: t \in \mathbb{R}\right\}$ be a real-valued random process on a probability space $(\Omega, \mathcal{F}, \mathcal{P})$. Our main interest in this paper is to study the almost sure behaviour of the normalized increments of the process $X$, namely

$$
Z_{\epsilon}(t)=\frac{X_{t+\epsilon}-X_{t}}{a(\epsilon)}
$$

for an appropriate normalizing function $a($.$) , when t$ varies in a bounded interval $I$ and $\epsilon \downarrow 0$. In classical cases there is no $a($.$) such that Z_{\epsilon}($.$) has almost surely a finite and non-zero$ limit pointwise or in $L^{p}(I, \lambda)(0<p \leq \infty), \lambda$ denoting Lebesgue measure. However, we shall prove that for a large family of processes we do have almost sure convergence in the sense that, for an appropriate choice of $a($.$) , the random measure \mu_{\epsilon}($.$) defined by$

$$
\mu_{\epsilon}(B)=\frac{1}{\lambda(I)} \lambda\left(\left\{t \in I, Z_{\epsilon}(t) \in B\right\}\right) ; \quad B \text { Borel set in } \mathbb{R},
$$

almost surely converges weakly to a measure $\mu^{*} \neq \delta_{0}$ as $\epsilon \downarrow 0$. Moreover, in various situations we shall have convergence of moments. We will prove results of this kind for:

(1) a class of Gaussian processes including fractional Brownian motion (for the standard Brownian motion the result is given by Wschebor 1992), stationary processes with certain local behaviour and some non-stationary ones (Section 2);

(2) processes with independent increments and symmetric stable law (Section 3);

(3) continuous martingales satisfying some regularity conditions (Section 4).

* To whom correspondence should be addressed.

1350-7265 (C) 1996 Chapman \& Hall 
For Gaussian processes and for martingales, one can obtain, as corollaries of our results, that the paths belong almost surely to a certain family of Besov spaces with critical parameter for which the Hölder property no longer holds.

The results will be proved in a more general context. Instead of $Z_{\epsilon}(t)$ as defined in (1) we put

$$
Z_{\epsilon}(t)=\frac{\epsilon \dot{X}_{\epsilon}(t)}{a(\epsilon)}
$$

where $\dot{X}_{\epsilon}$ is the derivative of $X_{\epsilon}$ and $X_{\epsilon}=\psi_{\epsilon} * X$ is a regularization of $X$ by means of convolution with an approximation of unity $\psi_{\epsilon}, \psi_{\epsilon}(t)=(1 / \epsilon) \psi(t / \epsilon), \epsilon>0, \psi$ a fixed function. Formula (1) corresponds to the special case $\psi=1_{[-1,0]}$.

In Section 5 we shall apply these methods to obtain almost sure weak approximations of the occupation measure of the process $X$ by means of the normalized number of crossings of $X_{\epsilon}$. A certain amount of work (Wschebor 1985; Azaïs 1989; Nualart and Wschebor 1991; Berzin and Wschebor 1993) has already been devoted to obtaining these kinds of approximation in $L^{p}(\Omega, \mathcal{F}, \mathcal{P})$ for each level of the process. Here the results are better in the sense that we obtain almost sure results and that we do not require the existence of the local time, but worse in the sense that the convergence holds as weak convergence of measures instead of pointwise convergence.

The paths of $X$ will be supposed to be càdlàg. The kernel function $\psi$ will be of bounded variation, with support included in $[-1,1], \int_{\mathbb{R}} \psi(t) \mathrm{d} t=1 . \Psi$ denotes the total variation measure of the (signed) measure with distribution function $\psi$. Let

$$
X_{\epsilon}(t)=\int_{-\infty}^{+\infty} \psi_{\epsilon}(t-s) X_{s} \mathrm{~d} s
$$

$C$ will denote a positive constant that may vary from line to line. $C_{\omega}$ will denote a positive random variable, almost surely finite, that may also vary from line to line. The parameter $\epsilon$ will be supposed to be bounded by some value $\epsilon_{0} . F_{\sigma}($.$) is the centred Gaussian distribution$ function with variance $\sigma^{2}$.

\section{Oscillation of Gaussian processes}

In this section, the interval $I$ is assumed for simplicity to be $[0,1]$, and $X_{t}$ is a centred Gaussian process with covariance function $r(t, s)$. We denote the incremental variance $V(s, t)=\operatorname{var}\left(X_{t}-X_{s}\right)$.

The theorems of this section rest on the following hypotheses:

$H_{1}$ : There exists a non-negative even function $a($.$) , non-decreasing on \mathbb{R}^{+}$, satisfying the following conditions:

$H_{1.1}: a($.$) is regularly varying at 0$ with exponent $\alpha, 0<\alpha<1$, that is:

$$
a(u)=u^{\alpha} L_{1}(u) \quad(u>0),
$$

where $L_{1}$ is slowly varying at zero (i.e. $\lim _{t \rightarrow 0} \frac{L_{1}(u t)}{L_{1}(t)}=1$ for all $u>0$ ). 
$H_{1.2}:$ For all $u, v \in[-1,1]$ and all $t \in[0,1]$,

$$
\frac{V(t+\epsilon u, t+\epsilon v)}{a^{2}(\epsilon(u-v))} \rightarrow b^{2}(t), \quad \epsilon \rightarrow 0,
$$

where the left-hand term is bounded above independently of $t, \epsilon, u$, $v$, hence $b($.$) is$ bounded and non-negative.

$H_{1.3}$ :

$$
a(2 \epsilon) \leq C a(\epsilon) .
$$

$\mathrm{H}_{2}$ : The covariance function $r(.,$.$) is twice continuously differentiable outside the$ diagonal and there exists a non-increasing slowly varying function $L_{2}$ at zero such that for some $\eta>0$ and for all $t$ and $s \in[-\eta, 1+\eta], t \neq s$,

$$
\left|\frac{\partial^{2} r(t, s)}{\partial t \partial s}\right| \leq C|t-s|^{2 \alpha-2} L_{2}(t-s) \text {. }
$$

$\alpha$ is the same as in $H_{1.1}$.

Define $C_{\psi}$ by

$$
C_{\psi}=\left[-\frac{1}{2} \int_{-1}^{1} \int_{-1}^{1}|u-v|^{2 \alpha} \mathrm{d} \psi(u) \mathrm{d} \psi(v)\right]^{\frac{1}{2}}
$$

Theorem 2.1. (General case). Suppose that the hypotheses $H_{1}$ and $H_{2}$ are satisfied. Define $Z_{\epsilon}(t)$ as in (2). Then as $\epsilon$ tends to zero,

(i) almost surely, for every integer $k>0$,

$$
\int_{I}\left(Z_{\epsilon}(t)\right)^{k} \mathrm{~d} t \rightarrow M_{k}=\mathrm{E}\left(\xi^{k}\right),
$$

where $\xi$ is a random variable with distribution function

$$
\int_{0}^{1} F_{C_{\psi} b(t)}(x) \mathrm{d} t
$$

(ii) almost surely, for every interval $J \subset I$,

$$
\lambda\left(\left\{t \in J / Z_{\epsilon}(t) \leq x\right\}\right) \rightarrow \int_{J} F_{C_{\psi} b(t)}(x) \mathrm{d} t, \quad x \neq 0 .
$$

Theorem 2.2. (Ordinary increments). Now define $Z_{\epsilon}(t)$ as in (1), and suppose that hypotheses $H_{1.1}$ and $H_{2}$ and the following hypothesis $H_{1.1}^{\prime}$ are satisfied:

$$
\begin{aligned}
& H_{1.1}^{\prime}: \text { For all } t \in[0,1], \\
& \qquad \begin{array}{l}
V(t, t+\epsilon) \\
V(t, t+\epsilon) \leq C(a(\epsilon))^{2} b^{2}(t) \quad \text { as } \epsilon \rightarrow 0
\end{array}
\end{aligned}
$$

Then the conclusions of Theorem 2.1 hold (with $C_{\psi}=1$ ). 
Theorem 2.3. (Stationary increments). We keep the same definition of $Z_{\epsilon}$ as in Theorem 2.2. Suppose that the process $X(t)$ has stationary increments, then $V(t, s)=\mathcal{V}(t-s), a(u)$ can be chosen equal to $(\mathcal{V}(u))^{1 / 2}$ and the function $b($.$) can be chosen to be identically one. The$ variable $\xi$ is a standard normal variable. If hypothesis $H_{2}$ is satisfied and if $(\mathcal{V}(\epsilon))^{1 / 2} \simeq$ $\epsilon^{\alpha} L_{1}(\epsilon)$ as $\epsilon \rightarrow 0, \alpha \in[0,1]$, then the conclusions of Theorem 2.1. hold (with $C_{\psi}=1$ ).

Several results about the family of processes $X_{\epsilon}(t)$ (in the general case) can be summarized in the following proposition.

Proposition 2.1. Suppose $H_{1}$ and $H_{2}$ are satisfied and $0<\epsilon<\eta\left(\eta\right.$ as in $\left.H_{2}\right)$; then

(a) $X_{\epsilon}(t)$ has almost surely $\mathcal{C}^{1}$ sample paths;

(b) $\operatorname{var}\left(\dot{X}_{\epsilon}(t)\right) \leq C \epsilon^{-2} a^{2}(\epsilon)$;

(c) $\operatorname{var}\left(Z_{\epsilon}(t)\right) \rightarrow C_{\psi}^{2} b^{2}(t)$ as $\epsilon$ tends to zero;

(d) for all $\gamma<\alpha$, and $t \in[0,1]$,

$$
\begin{aligned}
\left|\epsilon \dot{X}_{\epsilon}(t)\right| & \leq C_{\omega} \epsilon^{\gamma}, \\
\left|\epsilon \dot{X}_{\epsilon}(t)-\epsilon^{\prime} \dot{X}_{\epsilon^{\prime}}(t)\right| & \leq C_{\omega}\left|\epsilon-\epsilon^{\prime}\right|^{\gamma} ;
\end{aligned}
$$

(e) $|t-s|>3 \epsilon$ implies

$$
\operatorname{cov}\left(\dot{X}_{\epsilon}(t), \dot{X}_{\epsilon}(s)\right) \leq C(t-s)^{2 \alpha-2} L_{2}\left(\frac{|t-s|}{3}\right) .
$$

Proof of Theorem 2.1. Let $\epsilon>0$ and $k$ be a positive integer. We define the variable

$$
Y_{\epsilon}^{k}=\int_{0}^{1}\left(Z_{\epsilon}(t)\right)^{k} \mathrm{~d} t
$$

and first study its variance.

Proposition 2.1(b) implies that $Z_{\epsilon}(t)$ has a bounded variance, so

$$
\begin{aligned}
\operatorname{var}\left(Y_{\epsilon}^{k}\right) & =\int_{0}^{1} \int_{0}^{1} \operatorname{cov}\left(\left(Z_{\epsilon}(t)\right)^{k},\left(Z_{\epsilon}(s)\right)^{k}\right) \mathrm{d} t \mathrm{~d} s \\
& \leq C \epsilon+\iint_{|t-s|>3 \epsilon} \operatorname{cov}\left(\left(Z_{\epsilon}(t)\right)^{k},\left(Z_{\epsilon}(s)\right)^{k}\right) \mathrm{d} t \mathrm{~d} s .
\end{aligned}
$$

To bound the second term, note that there exist constants $C_{k, p}$ such that for every Gaussian centred vector $(U, W)$ and every $k=1,2, \ldots$, we have

$$
\operatorname{cov}\left(U^{k}, W^{k}\right)=\sum_{1 \leq p \leq k} C_{k, p}\left(\sigma_{u, w}\right)^{p}\left(\sigma_{u} \cdot \sigma_{w}\right)^{k-p},
$$

where $\sigma_{u}^{2}, \sigma_{w}^{2}$ and $\sigma_{u, w}$ are the variance of $U, W$ and the covariance of $(U, W)$, respectively. By Proposition 2.1(e), when $|t-s|>3 \epsilon$,

$$
\operatorname{cov}\left(Z_{\epsilon}(t), Z_{\epsilon}(s)\right) \leq C \epsilon^{2}(a(\epsilon))^{-2}(t-s)^{2 \alpha-2} L_{2}\left(\frac{|t-s|}{3}\right) .
$$


Thus, for $p \geq 1$,

$$
\iint_{|t-s|>3 \epsilon}\left(\operatorname{cov}\left(Z_{\epsilon}(t), Z_{\epsilon}(s)\right)\right)^{p} \mathrm{~d} t \mathrm{~d} s \leq C \epsilon^{2 p}(a(\epsilon))^{-2 p} \int_{\epsilon}^{1} u^{p(2 \alpha-2)}\left(L_{2}(u / 3)\right)^{p} \mathrm{~d} u .
$$

Now an elementary calculation shows that for every $\delta>0$ we have

$$
\iint_{|t-s|>3 \epsilon}\left(\operatorname{cov}\left(Z_{\epsilon}(t), Z_{\epsilon}(s)\right)\right)^{p} \mathrm{~d} t \mathrm{~d} s \leq C \epsilon^{1-\alpha-\delta} .
$$

Therefore

$$
\operatorname{var}\left(Y_{\epsilon}^{k}\right) \leq C \epsilon^{1-\alpha-\delta} .
$$

The Borel-Cantelli lemma now implies that

$$
Y_{\epsilon}^{k}-E\left(Y_{\epsilon}^{k}\right)
$$

converges almost surely along the sequence $\left\{\epsilon_{n}=n^{-a}, n \in \mathbb{N}\right\}$ for sufficiently large $a$. Now Proposition 2.1(b), (c) and the dominated convergence theorem imply that

$$
E\left(Y_{\epsilon}^{k}\right) \rightarrow E\left(\xi^{k}\right) \quad \text { as } \epsilon \rightarrow 0 .
$$

Thus $Y_{\epsilon_{n}}^{k}$ converges almost surely to $E\left(\xi^{k}\right)$.

We now study the behaviour of $Y_{\epsilon}^{k}$ for $\epsilon$ between two consecutive terms of the sequence $\left(\epsilon_{n}, n \in \mathbb{N}\right)$. Letting $\epsilon>0$, we define $n$ by $\epsilon_{n+1} \leq \epsilon<\epsilon_{n}$. Then

$$
\left|Y_{\epsilon}^{k}-Y_{\epsilon_{n}}^{k}\right| \leq\left|1-\frac{\left(a\left(\epsilon_{n}\right)\right)^{k}}{(a(\epsilon))^{k}}\right|\left|\int_{0}^{1}\left(Z_{\epsilon_{n}}(t)\right)^{k} \mathrm{~d} t\right|+(a(\epsilon))^{-k} \int_{0}^{1}\left|\left(\epsilon \dot{X}_{\epsilon}(t)\right)^{k}-\left(\epsilon_{n} \dot{X}_{\epsilon_{n}}(t)\right)^{k}\right| \mathrm{d} t .
$$

Since $a$ is monotone and regularly varying at zero, $\epsilon_{n} / \epsilon_{n+1} \rightarrow 1$ and $\int_{0}^{1}\left(Z_{\epsilon_{n}}(t)\right)^{k} \mathrm{~d} t$ is bounded, the first term tends to zero. As for the second, let $\gamma$ be as in Proposition 2.1(d).

$$
\begin{aligned}
\left(\epsilon \dot{X}_{\epsilon}(t)\right)^{k}-\left(\epsilon \dot{X}_{\epsilon}(t)+\left(\epsilon_{n} \dot{X}_{\epsilon_{n}}(t)-\epsilon \dot{X}_{\epsilon}(t)\right)\right)^{k} & \leq C_{\omega} \sum_{1 \leq p<k}\left(\begin{array}{l}
k \\
p
\end{array}\right) \epsilon^{\gamma p}\left(\epsilon_{n}-\epsilon\right)^{\gamma(k-p)} \\
& \leq C_{\omega} \epsilon^{\gamma(k-1)}\left(\epsilon_{n}-\epsilon\right)^{\gamma} .
\end{aligned}
$$

Now $\left|\epsilon_{n}-\epsilon\right| \leq C n^{-(a+1)}$ and $\epsilon_{n}=n^{-a}$, so that the second term in (3) is bounded by

$$
C_{\omega}\left(a\left(\epsilon_{n}\right)\right)^{-k} n^{-a \gamma(k-1)} n^{-(a+1) \gamma}
$$

which tends to zero if $\gamma$ is sufficiently close to $\alpha$. Relation (i) is then proved.

The moment convergence in (i) implies the weak convergence of measures in (ii) for the fixed interval $J=I$. This implies that this weak convergence holds simultaneously for all intervals $J$ with rational endpoints. Then (ii) follows from a density argument.

Proof of Proposition 2.1. (a) $\dot{X}_{\epsilon}(t)$ has the following Riemann-Stieltjes integral representation:

$$
\dot{X}_{\epsilon}(t)=\int_{-\infty}^{\infty} X_{t-u} \mathrm{~d} \psi_{\epsilon}(u) .
$$

The continuity of the paths of $X_{t}$ implies the result. 
(b)

$$
\begin{aligned}
\operatorname{var}\left(\dot{X}_{\epsilon}(t)\right) & =\epsilon^{-2} \iint_{\mathbb{R}^{2}} r(t-\epsilon u, t-\epsilon v) \mathrm{d} \psi(u) \mathrm{d} \psi(v) \\
& =\frac{-1}{2 \epsilon^{2}} \iint_{\mathbb{R}^{2}} V(t-\epsilon u, t-\epsilon v) \mathrm{d} \psi(u) \mathrm{d} \psi(v),
\end{aligned}
$$

since

$$
V(t, s)=r(t, t)+r(s, s)-2 r(t, s)
$$

and $\int \mathrm{d} \psi(u)=0$. Now use $H_{1.2}, H_{1.3}$ and the monotonicity of $a$.

(c)

$$
\operatorname{var}\left(Z_{\epsilon}(t)\right)=\frac{-1}{2 a^{2}(\epsilon)} \iint_{\mathbb{R}^{2}} V(t-\epsilon u, t-\epsilon v) \mathrm{d} \psi(u) \mathrm{d} \psi(v) .
$$

Use $H_{1.1}, H_{1.2}$ and the dominated convergence theorem.

(d) It is well known (Dudley 1973; Adler 1981) that for every $\gamma<\alpha, X(t)$ satisfies almost surely a Hölder condition with exponent $\gamma$ on the interval $[-\eta, 1+\eta]$. So if $\epsilon<\eta$ and $\epsilon^{\prime}<\eta$, then

$$
\begin{aligned}
\left|\epsilon \dot{X}_{\epsilon}(t)\right| & =\left|\int_{-\infty}^{+\infty}\left[X_{t-\epsilon u}-X_{t}\right] \mathrm{d} \psi(u)\right| \leq C_{\omega} \epsilon^{\gamma} \int_{-\infty}^{+\infty} u^{\gamma} \mathrm{d} \Psi(u)=C_{\omega} \epsilon^{\gamma}, \\
\left|\epsilon \dot{X}_{\epsilon}(t)-\epsilon^{\prime} \dot{X}_{\epsilon^{\prime}}(t)\right| & =\left|\int_{-\infty}^{+\infty} X_{t-\epsilon u}-X_{t-\epsilon^{\prime} u} \mathrm{~d} \psi(u)\right| \leq C_{\omega}\left|\epsilon-\epsilon^{\prime}\right|^{\gamma} .
\end{aligned}
$$

(e) Let $|t-s|>3 \epsilon$; then

$$
\begin{aligned}
\operatorname{cov}\left(\dot{X}_{\epsilon}(t), \dot{X}_{\epsilon}(s)\right) & =\iint_{\mathbb{R}^{2}} \psi(u) \psi(v) \frac{\partial^{2} r}{\partial s \partial t}[(t-\epsilon u, s-\epsilon v)] \mathrm{d} u \mathrm{~d} v \\
& \leq C \iint_{\mathbb{R}^{2}}|\psi(u)||\psi(v)||(t-s)-\epsilon(u-v)|^{2 \alpha-2} L_{2}(|(t-s)-\epsilon(u-v)|) \mathrm{d} u \mathrm{~d} v \\
& \leq C(t-s)^{2 \alpha-2} L_{2}\left(\frac{|t-s|}{3}\right)
\end{aligned}
$$

The proofs of Theorems 2.2 and 2.3 are essentially the same.

\section{Oscillation of stable processes with independent increments}

Now $\left\{X_{t}, t \in \mathbb{R}^{+}\right\}$is a symmetric $\alpha$-stable process with independent increments $(\alpha>1)$. It can be represented (Revuz and Yor 1991) as a 'subordinated Wiener process': there exist a standard Wiener process $W(t)$ and a homogenous independent $(\alpha / 2)$-stable subordinator $T_{t}$ such that

$$
X_{t}=W\left(T_{t}\right) .
$$


We define $\tilde{X}$ and $\tilde{T}$ as the extensions of $X$ and $T$ to $\mathbb{R}$ vanishing on $\mathbb{R}^{-}$. The processes $X_{\epsilon}$ are obtained by convolution of $\tilde{X}$ with the functions $\psi_{\epsilon}$. We define $a(\epsilon)$ by

$$
a(\epsilon)=\|\psi\|_{\alpha} \epsilon^{1 / \alpha},
$$

so that $Z_{\epsilon}(t)=\|\psi\|_{\alpha}^{-1} \epsilon^{1-1 / \alpha} \dot{X}_{\epsilon}(t)$.

The following lemma will be required in the proof of the main theorem of this section; its proof will follow after the proof of the theorem.

Lemma 3.1. For all real $\beta<1 / 2$,

$$
\sup _{\epsilon, \epsilon^{\prime} \in(0,1], \epsilon \neq \epsilon^{\prime}} \frac{\int_{0}^{1}\left|\epsilon \dot{X}_{\epsilon}(t)-\epsilon^{\prime} \dot{X}_{\epsilon^{\prime}}(t)\right| \mathrm{d} t}{\left|\epsilon-\epsilon^{\prime}\right|^{\beta}}<\infty
$$

almost surely.

Theorem 3.1. As $\epsilon$ tends to zero,

(i) for all $\lambda \in \mathbb{R}$,

$$
Y_{\epsilon}^{\lambda}=\int_{0}^{1} \mathrm{e}^{\mathrm{i} \lambda Z_{\epsilon}(t)} \mathrm{d} t \rightarrow E\left[\mathrm{e}^{\mathrm{i} \lambda X_{1}}\right]
$$

almost surely,

(ii) with probability one, for every bounded interval $J$ and all $x$,

$$
\lambda\left\{t \in J / Z_{\epsilon}(t) \leq x\right\} \rightarrow P\left[X_{1} \leq x\right] \lambda(J)
$$

(iii) with probability one, for every bounded interval $J$,

$$
\int_{J}\left|Z_{\epsilon}(t)\right| \mathrm{d} t \rightarrow E\left(\left|X_{1}\right|\right) \lambda(J) .
$$

Proof. (i) Let $g$ be a continuous function with compact support in $\mathbb{R}^{+}$and define

$$
Y=\int_{\mathbb{R}^{+}} g(t) X(t) \mathrm{d} t .
$$

Writing the integral as the limit of Riemann sums we get (Azaïs 1990)

$$
E\left(\mathrm{e}^{i Y}\right)=\exp \left(-\left(\int_{\mathbb{R}}\left(\int_{t}^{+\infty} g(u) \mathrm{d} u\right)^{\alpha} \mathrm{d} t\right)\right) .
$$

This implies that, for $t>\epsilon$ :

$$
Z_{\epsilon}(t) \stackrel{\stackrel{\mathrm{D}}{=}}{=} X_{1}
$$

In addition,

$$
\operatorname{var}\left[Y_{\epsilon}^{\lambda}\right]=\mathrm{E}\left(Y_{\epsilon}^{\lambda}-\mathrm{E}\left(\mathrm{e}^{\mathrm{i} \lambda \mathrm{X}_{1}}\right)\right)^{2}=\int_{0}^{1} \int_{0}^{1} \operatorname{cov}\left(\mathrm{e}^{\mathrm{i} \lambda Z_{\epsilon}(t)} \mathrm{e}^{\mathrm{i} \lambda Z_{\epsilon}(s)}\right) \mathrm{d} t \mathrm{~d} s .
$$


The independence of the increments of $X$ implies that, as soon as $|t-s|>2 \epsilon$, the integrand vanishes, so

$$
\operatorname{var}\left[\int_{0}^{1} \mathrm{e}^{\mathrm{i} \lambda Z_{\epsilon}(t)}\right] \leq 4 \epsilon
$$

This and the Borel-Cantelli lemma imply almost sure convergence of the function $Y_{\epsilon}^{\lambda}$ along the sequence

$$
\epsilon_{n}=n^{-a},
$$

for any fixed $a>1$. Relation (4) implies that the limit is $E\left[\mathrm{e}^{\mathrm{i} \lambda X_{1}}\right]$.

We now use Lemma 3.1. Let $\epsilon>0$, and define $n$ by

$$
\epsilon_{n+1} \leq \epsilon<\epsilon_{n} .
$$

Then

$$
\begin{aligned}
\left|Y_{\epsilon}^{\lambda}-Y_{\epsilon_{n}}^{\lambda}\right| & =\left|\int_{0}^{1} \mathrm{e}^{\mathrm{i} \lambda Z_{\epsilon}(t)}-\mathrm{e}^{\mathrm{i} \lambda Z_{\epsilon_{n}}(t)} \mathrm{d} t\right| \leq \lambda \int_{0}^{1}\left|Z_{\epsilon}(t)-Z_{\epsilon_{n}}(t)\right| \mathrm{d} t \\
& \leq C \lambda \frac{\left|\epsilon^{-1 / \alpha}-\epsilon_{n}^{-1 / \alpha}\right|}{\epsilon_{n}^{-1 / \alpha}}\left|Y_{\epsilon_{n}}^{\lambda}\right|+\lambda \epsilon^{-1 / \alpha} \int_{0}^{1}\left|\epsilon \dot{X}_{\epsilon}(t)-\epsilon_{n} \dot{X}_{\epsilon_{n}}(t)\right| \mathrm{d} t \\
& \leq C \lambda\left|1-\left(\epsilon / \epsilon_{n}\right)^{-1 / \alpha}\right|\left|Y_{\epsilon_{n}}^{\lambda}\right|+C_{\omega} \lambda \epsilon^{-1 / \alpha}\left|\epsilon-\epsilon_{n}\right|^{\beta} .
\end{aligned}
$$

Note that $\epsilon \simeq n^{-a}$ and $0 \leq \epsilon_{n}-\epsilon \leq C n^{-(a+1)}$, so that the first term tends to zero and the second is bounded by

$$
C_{\omega} \lambda n^{a / \alpha} n^{-\beta(a+1)}
$$

Since $\alpha>1, a$ and $\beta$ can be chosen sufficiently close to 1 and $\frac{1}{2}$ respectively, so that:

$$
a / \alpha-\beta(a+1)<0,
$$

and then relation (5) shows that $Y_{\epsilon}^{\lambda}-Y_{\epsilon_{n}}^{\lambda}$ tends almost surely to zero as $n \rightarrow+\infty$. So $Y_{\epsilon}^{\lambda}$ converges almost surely as $\epsilon \rightarrow 0$, and the proof of (i) is finished.

(ii) We have proved in (i) that for all $\lambda, Y_{\epsilon}^{\lambda}$ converges almost surely as $\epsilon$ tends to zero. By the Fubini theorem, for almost all $\omega, Y_{\epsilon}^{\lambda}$ tends to the function $E\left(\mathrm{e}^{\mathrm{i} \lambda X_{1}}\right)$ for almost every $\lambda$ as $\epsilon$ tends to zero.

The last assertion is equivalent by means of a standard modification of the Lévy-Cramér theorem to the weak convergence of the associated probability. The rest of the proof is identical to that of Theorem 2.1.

(iii) Using the same separability argument as in the proof of Theorem 2.1, we see that it suffices to prove the result for the case $J=[0,1]$. Denote by $N(\mathrm{~d} x)$ the Lévy measure of the stable process $X: N(\mathrm{~d} x)=(\alpha / 2)|x|^{-(\alpha+1)} \mathrm{d} x$. Let $\gamma$ (to be made more precise later on) be such that $0 \leq \gamma<1 / \alpha$. For all $\epsilon>0$, we define the decomposition

$$
X(t)=\int_{|x|<\epsilon^{\gamma}} x \nu_{t}^{*}(\mathrm{~d} x)+\int_{|x| \geq \epsilon^{\gamma}} x \nu_{t}^{*}(\mathrm{~d} x)=X_{t}^{1, \epsilon}+X_{t}^{2, \epsilon},
$$


where $\nu_{t}^{*}(B)=\nu_{t}(B)-t N(B), B \subset\{x:|x|>a\}$ for some $a>0$, and $\nu_{t}($.$) is the Poisson$ measure of the discontinuities of the process. The first term in (6) is a Wiener integral with respect to the random set function $\nu_{t}^{*}($.$) . The second term is given by a finite sum and X_{t}^{1, \epsilon}$ and $X_{t}^{2, \epsilon}$ are independent Lévy processes. We first give the proof in the case of ordinary increments, i.e.

$$
Z_{\epsilon}(t)=\frac{X_{t+\epsilon}-X_{t}}{\epsilon^{1 / \alpha}}
$$

and conclude by giving the modifications for the general case. The decomposition corresponding to (6) for $Z_{\epsilon}(t)$ is denoted by

$$
Z_{\epsilon}(t)=Z_{\epsilon}^{(1)}(t)+Z_{\epsilon}^{(2)}(t) .
$$

Let $\|\cdot\|_{1}$ be the norm of $L^{1}([0,1], \lambda)$. Since $X_{t}^{2, \epsilon}$ is a step function,

$$
\left\|Z_{\epsilon}^{(2)}(.)\right\|_{1} \leq \epsilon^{1-1 / \alpha} \int_{|u| \geq \epsilon^{\gamma}}|u| \nu_{1+\epsilon_{0}}(\mathrm{~d} u) .
$$

By the law of large numbers,

$$
\int_{|u| \geq \epsilon^{\gamma}}|u| \nu_{1+\epsilon_{0}}(\mathrm{~d} u) \simeq\left(1+\epsilon_{0}\right) \int_{|u| \geq \epsilon^{\gamma}}|u| N(\mathrm{~d} u)=C \epsilon^{\gamma(1-\alpha)},
$$

thus

$$
\left\|Z_{\epsilon}^{(2)}(.)\right\|_{1} \leq C \epsilon^{((\alpha-1) / \alpha)(1-\gamma \alpha)} \rightarrow 0 .
$$

So it suffices to study $Z_{\epsilon}^{(1)}(t)$.

$$
E\left(\left(Z_{\epsilon}^{(1)}(t)\right)^{2}\right)=C \epsilon^{1-2 / \alpha} \int_{|x|<\epsilon^{\gamma}} x^{2} N(\mathrm{~d} x)=C \epsilon^{(\alpha-2)(1 / \alpha-\gamma)} .
$$

We calculate the variance

$$
\operatorname{var}\left(\int_{0}^{1}\left|Z_{\epsilon}^{(1)}(t)\right| \mathrm{d} t\right)=\int_{0}^{1} \int_{0}^{1} \operatorname{cov}\left(\left|Z_{\epsilon}^{(1)}(t)\right|,\left|Z_{\epsilon}^{(1)}(s)\right|\right) \mathrm{d} t \mathrm{~d} s .
$$

The integrand vanishes as soon as $|t-s|>\epsilon$ and is bounded using (8) otherwise. We have

$$
\operatorname{var}\left(\int_{0}^{1}\left|Z_{\epsilon}^{(1)}(t)\right| \mathrm{d} t\right) \leq C \epsilon^{1+(\alpha-2)(1 / \alpha-\gamma)} .
$$

Relations (4) and (7) imply

$$
\mathrm{E}\left(\int_{0}^{1}\left|Z_{\epsilon}^{(1)}(t)\right| \mathrm{d} t\right) \rightarrow \mathrm{E}\left|X_{1}\right| .
$$

As in part (i) of the proof, for $\epsilon_{n}=n^{-a}$ with $a>(1+(\alpha-2)(1 / \alpha-\gamma))^{-1}$ almost surely,

$$
\int_{0}^{1}\left|Z_{\epsilon_{n}}^{(1)}(t)\right| \mathrm{d} t \rightarrow \mathrm{E}\left|X_{1}\right| \quad(n \rightarrow \infty) .
$$

Relation (7) implies that the same relation is true putting $Z_{\epsilon_{n}}(t)$ instead of $Z_{\epsilon_{n}}^{(1)}(t)$. For 
$\epsilon_{n+1} \leq \epsilon<\epsilon_{n}$, using Lemma 3.1 with $0<\beta<\frac{1}{2}$, we have

$$
\left|\int_{0}^{1}\right| Z_{\epsilon}(t)\left|\mathrm{d} t-\int_{0}^{1}\right| Z_{\epsilon_{n}}(t)|\mathrm{d} t| \leq C_{\omega}\left[\epsilon^{-1 / \alpha}\left|\epsilon-\epsilon_{n}\right|^{\beta}+\left(\left(\epsilon_{n} / \epsilon\right)^{1 / \alpha}-1\right) \int_{0}^{1}\left|Z_{\epsilon_{n}}(t)\right| \mathrm{d} t\right] .
$$

The second term tends to zero almost surely, since $\int_{0}^{1}\left|Z_{\epsilon_{n}}\right| \mathrm{d} t$ is bounded. The first is bounded by

$$
C_{\omega} n^{a / \alpha} n^{-\beta(a+1)},
$$

which tends to zero as $n \rightarrow \infty$ if we have chosen $\beta$ close enough to $\frac{1}{2}, \gamma$ to $1 / \alpha$ and $a$ to $(1+(\alpha-2)(1 / \alpha-\gamma))^{-1}$ so that $a / \alpha-(a+1) \beta<0$, which is feasible. This ends the proof for ordinary increments.

For general increments we have to remark that

$$
Z_{\epsilon}^{(i)}(t)=\int \frac{X_{t-\epsilon u}^{i, \epsilon}-X_{t}^{i, \epsilon}}{(\epsilon|u|)^{1 / \alpha}}|u|^{1 / \alpha} \mathrm{d} \psi(u),
$$

to get the same bounds (7) and (8) and to pass to the limit as $\epsilon \rightarrow 0$ in $\int_{0}^{1}\left|Z_{\epsilon}\right| \mathrm{d} t$ in a similar way.

Proof of Lemma 3.1. Let $0<\beta<\frac{1}{2}$. On the interval $\left[0, T_{1+\epsilon}\right]$ the Wiener process satisfies almost surely a Hölder condition with exponent $\beta$ :

$$
|W(s)-W(t)| \leq C_{\omega}|t-s|^{\beta} \quad t, s \in\left[0, T_{1+\epsilon}\right] .
$$

We remark that $\epsilon \dot{X}_{\epsilon}(t)$ is given by

$$
\epsilon \dot{X}_{\epsilon}(t)=\int_{-\infty}^{+\infty} \tilde{X}_{t-\epsilon u} \mathrm{~d} \psi(u),
$$

so that

$$
\int_{0}^{1}\left|\epsilon \dot{X}_{\epsilon}(t)-\epsilon^{\prime} \dot{X}_{\epsilon^{\prime}}(t)\right| \mathrm{d} t \leq \int_{-\infty}^{+\infty} \int_{0}^{1}\left|\tilde{X}_{t-\epsilon u}-\tilde{X}_{t-\epsilon^{\prime} u}\right| \mathrm{d} t \mathrm{~d} \Psi(u) .
$$

By the Jensen inequality

$$
\begin{aligned}
\left(\int_{0}^{1}\left|\tilde{X}_{t-\epsilon u}-\tilde{X}_{t-\epsilon^{\prime} u}\right| \mathrm{d} t\right)^{1 / \beta} & \leq \int_{0}^{1}\left|\tilde{X}_{t-\epsilon u}-\tilde{X}_{t-\epsilon^{\prime} u}\right|^{1 / \beta} \mathrm{d} t \\
& \leq C_{\omega} \int_{0}^{1}\left|\tilde{T}_{t-\epsilon u}-\tilde{T}_{t-\epsilon^{\prime} u}\right| \mathrm{d} t \\
& \leq C_{\omega}\left|\left(\epsilon-\epsilon^{\prime}\right) u\right| T_{1+\epsilon_{0}}=C_{\omega}\left|\left(\epsilon-\epsilon^{\prime}\right) u\right|,
\end{aligned}
$$

since $T$ is non-decreasing, $T_{0}=0$. Thus

$$
\int_{0}^{1}\left|\epsilon \dot{X}_{\epsilon}(t)-\epsilon^{\prime} \dot{X}_{\epsilon^{\prime}}(t)\right| \mathrm{d} t \leq C_{\omega} \int_{-\infty}^{+\infty}\left(\epsilon-\epsilon^{\prime}\right)^{\beta} u^{\beta} \mathrm{d} \Psi(u)=C_{\omega}\left(\epsilon-\epsilon^{\prime}\right)^{\beta} .
$$




\section{Oscillation of martingales}

We consider similar results for Itô integrals $\left\{X_{t}: t \geq 0\right\}$ of the form

$$
X_{t}=\int_{0}^{t} b(s) \mathrm{d} W_{s}
$$

where $\{b(t): t \geq 0\}$ is adapted to the filtration generated by the Wiener process $\left\{W_{t}: t \geq 0\right\}$ and $b($.$) is locally essentially bounded. We extend X, W$ and $b$ by 0 on $\mathbb{R}^{-}$. Define $Z_{\epsilon}(t)$ as $\left(\sqrt{\epsilon} /\|\psi\|_{2}\right) \dot{X}_{\epsilon}(t)$, so that $a(\epsilon)=\|\psi\|_{2} / \sqrt{\epsilon}$.

Theorem 4.1. Almost surely, for every bounded interval $J$

$$
\lambda\left(\left\{t: t \in J, Z_{\epsilon}(t) \leq x\right\}\right) \rightarrow \int_{J} F_{b(t)}(x) \mathrm{d} t \quad \text { as } \epsilon \rightarrow 0, x \neq 0 .
$$

Proof. Without loss of generality we suppose that $J \subset[0,1]$ and that

$$
\|b(.)\|_{\infty} \leq M,
$$

where $M$ is a non-random constant and where the norm is taken in $L^{\infty}\left(\left[0,1+\epsilon_{0}\right]\right)$.

Check that for all $t, 0 \leq t \leq 1$,

$$
\epsilon^{1 / 2} \dot{X}_{\epsilon}(t)=\epsilon^{1 / 2} \dot{W}_{\epsilon}(t) b(t-\epsilon)+\epsilon^{1 / 2} \int_{t-\epsilon}^{t+\epsilon} \psi_{\epsilon}(t-s)(b(s)-b(t-\epsilon)) \mathrm{d} W_{s} .
$$

Almost surely, the distribution of the first term on the right-hand side of (10), considered as a function of $t$ in the measure space $([0,1], \lambda)$, tends to that on the right-hand side of (9) see Section 2 or Wschebor (1992) and take into account the continuity of the translation operator on $L^{2}([0,1], \lambda)$. To prove (9), it will be enough to show that the second term in the right-hand side of (10) almost surely tends to zero in $L^{2}([0,1], \lambda)$.

Put

$$
\begin{aligned}
R_{\epsilon}(t) & =\epsilon^{1 / 2} \int_{t-\epsilon}^{t+\epsilon} \psi_{\epsilon}(t-s)(b(s)-b(t-\epsilon)) \mathrm{d} W_{s} \\
\Delta_{\epsilon} & =\mathrm{E}\left\{\left[\int_{0}^{1}\left[R_{\epsilon}(t)\right]^{2} \mathrm{~d} t-\epsilon \int_{0}^{1} \mathrm{~d} t \int_{t-\epsilon}^{t+\epsilon} \psi_{\epsilon}^{2}(t-s)(b(s)-b(t-\epsilon))^{2} \mathrm{~d} s\right]^{2}\right\} \\
& =\epsilon^{2} \mathrm{E}\left\{\int_{0}^{1} \int_{0}^{1} Y_{\epsilon}(t) Y_{\epsilon}(s) \mathrm{d} t \mathrm{~d} s\right\},
\end{aligned}
$$

with

$$
Y_{\epsilon}(t)=\left(\int_{t-\epsilon}^{t+\epsilon} \psi_{\epsilon}(t-u)(b(u)-b(t-\epsilon)) \mathrm{d} W_{u}\right)^{2}-\int_{t-\epsilon}^{t+\epsilon} \psi_{\epsilon}^{2}(t-u)(b(u)-b(t-\epsilon))^{2} \mathrm{~d} u .
$$

It is clear that if $|t-s| \geq 2 \epsilon$, then $\mathrm{E}\left[Y_{\epsilon}(t) Y_{\epsilon}(s)\right]=0$, so that

$$
\Delta_{\epsilon} \leq C \epsilon \text {, }
$$


where the constant $C$ depends upon $M$ and $\|\psi\|_{2}$. Relation (11) follows from the Burkholder-Davis-Gundy inequality (Karatzas and Shreve 1988), the fact that $M$ is an upper bound for $\|b\|_{\infty}$ and

$$
\int_{t-\epsilon}^{t+\epsilon} \psi_{\epsilon}^{2}(t-s) \mathrm{d} s=\frac{1}{\epsilon}\|\psi\|_{2}^{2} .
$$

From (11) and the Borel-Cantelli lemma, we conclude that almost surely

$$
\begin{gathered}
\int_{0}^{1}\left(R_{\epsilon_{n}}(t)\right)^{2} \mathrm{~d} t-\epsilon_{n} \int_{0}^{1} \mathrm{~d} t \int_{t-\epsilon_{n}}^{t+\epsilon_{n}} \psi_{\epsilon_{n}}(t-s)\left(b(s)-b\left(t-\epsilon_{n}\right)\right)^{2} \mathrm{~d} s \underset{n \rightarrow+\infty}{\longrightarrow} 0 \\
\epsilon_{n}=n^{-a} \quad n=1,2 \ldots ; a>1 .
\end{gathered}
$$

Note also that almost surely

$$
\begin{aligned}
\left.\epsilon \int_{0}^{1} \mathrm{~d} t \int_{t-\epsilon}^{t+\epsilon} \psi_{\epsilon}^{2}(t-s)(b(s)-b(t-\epsilon))\right)^{2} \mathrm{~d} s & =\int_{0}^{1} \mathrm{~d} t \int_{-1}^{1} \psi^{2}(v)(b(t-\epsilon v)-b(t-\epsilon))^{2} \mathrm{~d} v \\
& =\int_{-1}^{1} \psi^{2}(v)\left\|b(.)-b_{\epsilon(v-1)}(.)\right\|_{2}^{2} \mathrm{~d} v
\end{aligned}
$$

where $b_{\epsilon(v-1)}(z)=b(z+\epsilon(v-1))$. Using the bounded convergence theorem, the expression above tends to zero. This, together with (12), implies that almost surely

$$
\int_{0}^{1}\left(R_{\epsilon}(t)\right)^{2} \mathrm{~d} t \rightarrow 0,
$$

on the sequence $\epsilon=\epsilon_{n}$. To prove that (13) holds without restriction we use the bound for all $\eta>0$,

$$
\begin{aligned}
\left|\epsilon \dot{X}_{\epsilon}(t)-\epsilon^{\prime} \dot{X}_{\epsilon^{\prime}}(t)\right| & =\left|\int_{-1}^{1} X_{t-\epsilon u} \mathrm{~d} \psi_{u}-\int_{-1}^{1} X_{t-\epsilon^{\prime} u} \mathrm{~d} \psi_{u}\right| \\
& \leq C \sup _{|u|<1}\left|X_{t-\epsilon u}-X_{t-\epsilon^{\prime} u}\right|<C_{\omega}\left|\epsilon-\epsilon^{\prime}\right|^{\frac{1}{2}-\eta} .
\end{aligned}
$$

This finishes the proof of the theorem.

Note that the previous computations show that for every bounded interval $J$

$$
\int_{J}\left|Z_{\epsilon}(t)\right|^{2} \mathrm{~d} t
$$

is almost surely bounded for $\epsilon>0$, which implies the uniform integrability of $\left\{Z_{\epsilon}(.) ; \epsilon>0\right\}$ in $L^{1}(J, \lambda)$. So using Theorem 4.1 one obtains that almost surely

$$
(1 / \lambda(J)) \int_{J}\left|Z_{\epsilon}(t)\right| \mathrm{d} t \rightarrow \mathrm{E}(|\xi|),
$$

$\xi$ being a random variable with distribution function

$$
(1 / \lambda(J)) \int_{J} F_{b(t)}(x) \mathrm{d} t .
$$




\section{Almost sure weak approximation of the occupation measure}

Let $g(t)$ be a real function defined over an interval $I$. We define the occupation measure $\Gamma_{g}$ by

$$
\Gamma_{g}(I \times B)=\lambda\{t \in I, g(t) \in B\},
$$

where $I$ and $B$ are Borel sets on the real line; and the number of crossings of level $u, N_{u}(g, I)$ by

$$
N_{u}(g, I)=\#\{t \in I, g(t)=u\} .
$$

Theorem 5.1. With the hypotheses and notations stated in Theorems 2.1, 2.2, 2.3, 3.1 and 4.1, almost surely, for every continuous real function $f$ and every bounded interval $J$, we have

$$
\theta \epsilon(a(\epsilon))^{-1} \int_{-\infty}^{+\infty} f(u) N_{u}\left(X_{\epsilon}, J\right) \mathrm{d} u \underset{\epsilon \rightarrow 0}{\longrightarrow} \int_{J} \int_{\mathbb{R}} f(u)|b(t)| \Gamma_{X}(\mathrm{~d} t, \mathrm{~d} u)
$$

where $\theta$ is a constant given by

$$
\theta= \begin{cases}(\pi / 2)^{1 / 2}\left(C_{\psi}\right)^{-1} & \text { for Gaussian processes } \\ (\pi / 2)^{1 / 2} & \text { for martingales } \\ (\mathrm{E}|X(1)|)^{-1} & \text { for stable processes }\end{cases}
$$

For stable processes, the function $b(t)$ is identically equal to 1.

Proof. We divide the proof into several steps. First, we use the following equality (Nualart and Wschebor 1991).

For continuous $f, \mathbb{R} \rightarrow \mathbb{R}$ and $g$ of class $\mathcal{C}^{1}, \mathbb{R} \rightarrow \mathbb{R}$, we have

$$
\int_{-\infty}^{+\infty} f(u) N_{u}(g, J) \mathrm{d} u=\int_{J} f[g(t)]\left|g^{\prime}(t)\right| \mathrm{d} t .
$$

Next, we write

$$
\begin{aligned}
\theta \epsilon(a(\epsilon))^{-1} \int_{\mathbb{R}} f(u) N_{u}\left(X_{\epsilon}, J\right) \mathrm{d} u & =\theta \int_{J} f\left(X_{\epsilon}(t)\right)\left|Z_{\epsilon}(t)\right| \mathrm{d} t \\
& =\theta \int_{J} f\left(X_{\epsilon}(t)\right)-f\left(X(t) \mid Z_{\epsilon}(t)\right)\left|\mathrm{d} t+\theta \int_{J} f\left(X_{t}\right)\right| Z_{\epsilon}(t) \mid \mathrm{d} t .
\end{aligned}
$$

We study the first term in (15). Note that in each case we have proved that $\int_{J}\left|Z_{\epsilon}(t)\right| \mathrm{d} t$ is almost surely bounded. Over $J, X_{\epsilon}(t)$ and $X(t)$ are almost surely uniformly bounded and $X(t)$ is almost everywhere continuous, so $f\left(X_{\epsilon}(t)\right)-f(X(t))$ converges almost everywhere to zero. Thus the first term tends to zero.

Let us look at the second term in (15). For each interval $J$ we know that, as $\epsilon$ tends to zero,

$$
\theta \int_{J}\left|Z_{\epsilon}(t)\right| \mathrm{d} t \rightarrow \int_{J}|b(t)| \mathrm{d} t
$$


almost surely; this follows from weak convergence (Theorems 2.1, 2.2, 2.3, 3.1(i) and 4.1) plus uniform integrability of the family $\left\{Z_{\epsilon}(.) ; \epsilon>0\right\}$ in the Gaussian and martingale cases, and from the direct proof (Theorem 3.1(iii)) in the stable case. Now a density argument shows that (16) holds true simultaneously for all bounded intervals $J$. Hence, for every function $g: J \rightarrow \mathbb{R}$ which can be written as a uniform limit of linear combinations of indicator functions of intervals, we have, almost surely,

$$
\theta \int_{J} g(t)\left|Z_{\epsilon}(t)\right| \mathrm{d} t \underset{\epsilon \rightarrow 0}{\longrightarrow} \int_{J} g(t)|b(t)| \mathrm{d} t .
$$

That is the case for $g(t)=f\left(X_{t}\right)$ if $f$ is continuous since $t \rightarrow X_{t}$ is càdlàg. The equality

$$
\int_{J} f\left(X_{t}\right)|b(t)| \mathrm{d} t=\int_{J} \int_{\mathbb{R}} f(u)|b(t)| \Gamma_{X}(\mathrm{~d} t, \mathrm{~d} u)
$$

is obvious from the definition of $\Gamma_{X}$. This completes the proof.

\section{References}

Adler, R.J. (1981) The Geometry of Random Fields. New York: Wiley.

Azaïs, J.-M. (1989) Approximation des trajectoires et temps local des diffusions. Ann. Inst. H. Poincaré, Probab. Statist, 25, 175-194.

Azaïs, J.-M. (1990) Conditions for convergence of number of crossings to the local time. Application to stable processes with independent increments and to Gaussian processes. Probab. Math. Statist., 11, 19-36.

Berzin, C. and Wschebor, M. (1993) Approximation du temps local des surfaces gaussiennes. Probab. Theory Related Fields, 96, 1-32.

Dudley, R.M. (1973) Sample functions of the Gaussian process. Ann. Probab., 1, 66-103.

Karatzas, I. and Shreve, S.E. (1988) Brownian Motion and Stochastic Calculus. Berlin: Springer-Verlag. Nualart, D. and Wschebor, M. (1991) Integration par parties dans l'espace de Wiener et approximation du temps local. Probab. Theory Related Fields, 90, 83-109.

Revuz, R. and Yor, M. (1991) Continuous Martingales and Brownian Motion. Berlin: Springer-Verlag. Wschebor, M. (1985) Surfaces Aléatoires, Mesure Géométrique des Ensembles de Niveau. Lecture Notes in Math. 1147. Berlin: Springer-Verlag.

Wschebor, M. (1992) Sur les accroissements du processus de Wiener. C.R. Acad. Sci. Paris (1), 315, $1293-1296$.

Received April 1994 and revised January 1996 